\title{
On Initial Segments of Turing Degrees Containing Simple T-Mitotic but not $w t t$-Mitotic Sets
}

\author{
Arsen H. Mokatsian \\ Institute for Informatics and Automation Problems of NAS RA \\ e-mail: arsenmokatsian@gmail.com
}

\begin{abstract}
We consider the properties of computably enumerable (c.e.) Turing degrees containing sets, which possess the property of a $T$-mitotic splitting but don't have a $w t t$-mitotic splitting.

It is proved that for any noncomputable c.e. degree $\boldsymbol{b}$ there exists a degree $\boldsymbol{a}$, such that $\boldsymbol{a} \leq \boldsymbol{b}$ and $\boldsymbol{a}$ contains a simple $T$-mitotic set, which is not $w t$-mitotic.
\end{abstract}

Keywords: Mitotic set, T-reducibility, wtt-reducibility, Simple set, Contiguous degree.

\section{Introduction}

We shall use the notions and terminology introduced in Soare [1], Rogers [2].

Notations.

We deal with sets and functions over the nonnegative integers $\omega=\{0,1,2, \ldots\}$.

Let $\omega_{e v}=\{x:(\exists k)(x=2 k)\} ; \omega_{o d}=\{x:(\exists k)(x=2 k+1)\}$.

Let $\varphi_{e}$ be the $e^{t h}$ partial computable function in the standard listing (Soare [1, p. 15, p. 25]).

If $A \subseteq \omega$ and $e \in \omega$, let $\Phi_{e}^{A}(x)=\Phi_{e}(A: x)=\{e\}^{A}(x)$ (see Soare [1, pp. 48-50]).

$\chi_{A}$ denotes the characteristic function of $A$, which is often identified with $A$ and written simply as $A(x)$.

Let $\varphi(x) \downarrow$ denotes that $\varphi(x)$ is defined, $\varphi(x) \uparrow$ denotes that $\varphi(x)$ is undefined.

$W_{e}=\operatorname{dom} \varphi_{e}=\left\{x: \varphi_{x}(x) \downarrow\right\}$.

$\varphi_{e, a t s+1}(x) \downarrow$ denotes $\varphi_{e, s+1}(x) \downarrow \& \varphi_{e, s}(x) \uparrow$.

$x \in W_{e, a t s+1}$ denotes $x \in W_{e, s+1}-W_{e, s}$.

In the literature, Turing reducibility is usually taken as the main reducibility. If the word "reducibility" is used without a further explanation, it means, as a rule, Turing reducibility. If the term "degree of unsolvability" is used without a further explanation, the $T$-degree is usually meant.

Definition 1: The use function $u(A ; e, x, s)$ is $1+$ (the maximum number used in computation if $\left.\Phi_{e s}^{A}(x) \downarrow\right)$, and $=0$, otherwise. The use function $u(A ; e, x)$ is $u(A ; e, x, s)$ if $\Phi_{e s}^{A}(x) \downarrow$ for some $s$, and is undefined if $\Phi_{e s}^{A}(x) \uparrow$. 
Definition 2: $A$ is computable in (Turing reducible to) $B$, written $A \leq_{T} B$, if $A=\Phi_{e}^{B}$ for some $e$ (Soare $[1$, p. 50]).

Definition 3: $A$ is weak truth-table reducible to $B$, written $A \leq_{w t t} B$, if $(\exists e)\left[A=\Phi_{e}^{B} \&(\exists\right.$ computable $f)(f(x) \geq u(B ; e, x))]$ (where $u(B ; e, x)$ is the use function from Definition 1) (Rogers [2, p. 158]).

Definition 4: If $A$ is a noncomputable computably enumerable (c.e.) set, then a splitting of $A$ is a pair $A_{1}, A_{2}$ of disjoint c.e. sets such that $A_{1} \cup A_{2}=A$ (Downey, Stob [3, p. 4]).

Definition 5: C.e. set $A$ is T-mitotic (wtt-mitotic), if there is a splitting $A_{1}, A_{2}$ of $A$ such that $A_{1} \equiv_{T} A_{2} \equiv_{T} A\left(A_{1} \equiv_{w t t} A_{2} \equiv_{w t t} A\right)$ (Downey, Stob [3, p. 83]).

Definition 6: (i) $A$ set is immune, if it is infinite but contains no infinite c.e. set.

(ii) $A$ set is simple, if $A$ is c.e. and $\bar{A}$ is immune (Soare [1, p. 78]).

Definition 7: $A$ c.e. degree $\boldsymbol{a}$ is contiguous if for every pair $A, B$ of c.e. sets in $\boldsymbol{a}, A \equiv_{w t t} B$ (Downey, Stob [3, p. 45]).

Note that each contiguous degree, by definition, doesn't contain $T$-mitotic sets, which are not $w t t$-mitotic.

Lachlan proved the existence of nonmitotic c.e. set (Lachlan [4]).

Ladner proved the existence of completely mitotic c.e. degree (Ladner [5]).

Ladner and Sasso [6] proved, that for every nonzero c.e. degree $\boldsymbol{b}$ there is a nonzero c.e. degree $\boldsymbol{a} \leq \boldsymbol{b}$ such that $\boldsymbol{a}$ is contiguous (see also Downey, Stob [3]).

Thus, there is an infinite class of contiguous degrees, and these degrees, as we have mentioned, don't contain $T$-mitotic sets, which are not wtt-mitotic.]

Ingrassia ([7]) proved the density of nonmitotic c.e. sets (in $\boldsymbol{R}$ ) (see also Downey, Sla$\operatorname{man}[8])$.

E. J. Griffiths ([9]) proved the following Theorem: There exists a low c.e. degree $\boldsymbol{u}$ such that if $\boldsymbol{v}$ is a c.e. degree and $\boldsymbol{u} \leq \boldsymbol{v}$, then $\boldsymbol{v}$ is not completely mitotic.

\section{Preliminaries, Basic Modules}

Theorem 1: For any noncomputable c.e. degree $\boldsymbol{b}$ there is a degree $\boldsymbol{a}$ such that $\boldsymbol{a} \leq \boldsymbol{b}$ and a contains a simple T-mitotic, but not wtt-mitotic set.

Proof. (sketch) Let $h$ be a general computable function that maps $\omega$ to $\omega^{2}$. Let $\left(\Psi_{i}, \psi_{i}\right)$ denotes the pair $\left(\Phi_{i_{0}}, \varphi_{i_{0}}\right)$ for all $i$, where $h(i)=\left(i_{0}, i_{1}\right)$ (note that $\Psi_{i}$ is wtt-reduction with $\psi_{i}$, denoting the corresponding use function).

It is known (Ladner [10]) that the computably enumerable set $A$ is $T$-mitotic, $\Leftrightarrow A$ is $T$-autoreducible, and similarly, the computably enumerable set $A$ is wtt-mitotic, $\Leftrightarrow A$ is $w t$-autoreducible (Downey, Stob [3], see also Trakhtenbrot [11]).

Therefore, in order to achieve non-wtt-mitoticity, it is enough for us to achieve non-wttautoreducibility.

Thus, to prove our theorem, it is necessary to construct such a c.e. set $A$, so that the following requirements are met. 
$R_{e}:(\exists x) \neg\left(\Psi_{e}(A \bigcup\{x\} ; x)=A(x)\right)$, if $(\forall z \leq y)\left(\psi_{e}(z) \downarrow\right)$.

$P_{e}:\left(W_{e}\right.$ is infinite $) \Rightarrow(\exists x)\left(x \in W_{e} \& x \in A\right)$.

Note that satisfying the $R_{e}$ requirement (for all $e$ ) provides the infinity of the set $\bar{A}$.

Order the requirements in the following priority ranking: $R_{0}, P_{0}, R_{1}, P_{1}, \ldots, R_{n}, P_{n}, \ldots$

Let $l(e, s)=\max \left\{x:(\forall y<x)\left(\Psi_{e, s}\left(A_{s} \cup\{y\}: y\right)=A(y) \&(\forall z \leq x)\left(\Psi_{e}(z) \downarrow\right)\right)\right\}$.

The main strategy for satisfying $R_{e}$ is the following: we select a number (the so-called follower) $x$ (which should be accompanied by two more elements $x-2$ and $x-1$, and possibly, the third - $\hat{x}$; an exact definition of the attendant numbers of the follower $x$, namely $(x-2)$, $(x-1), \hat{x}$, will be given hereinafter), we wait until $l(e, s)>x$ and enumerate $x$ in $A_{s+1}$, if $(\forall z<x)\left(\psi_{e, s}(z) \downarrow\right)$, setting $r(e, s+1)=u(x, e, s)$, where $u(x, e, s)=u\left(\Psi_{e, s}\left(A_{s} \bigcup\{x\} ; x\right)\right)$. An $B$-permitting procedure is introduced in order to provide $A \leq_{T} B$ (where $B$ is a c.e. set from degree $\boldsymbol{b}$ ).

To satisfy the requirement of $R_{e}$ at each stage, we have a finite set of followers $x_{1, s},<\ldots<$ $x_{n, s}$. In this construction, a modification of the $B$-permitting method is used. We treat the interval $[0, \ldots, \mathrm{i}]$ as allowing for $x_{i, s}$.

To satisfy the requirement of $P_{e}$ at each stage, we have a finite set of followers $y_{1, s},<\ldots<$ $y_{n, s}$. For requirement $P_{e}$, the usual $B$-permitting method is used.

The construction will be such that if eventually we have $\Psi_{e}(A \cup\{x\} ; x)=A(x) \& \psi_{e}$ is a total function, then it will be possible to compute $B$.

The ground of satisfactions for requirements of $R_{e}$ and $P_{e}$ will be given below.

\subsection{Basic Module for $R_{e}$}

The followers $x_{1, s}, \ldots, x_{n, s}$ satisfy the following rules below.

Appointment. If $x_{i, s}$ is currently defined and $x_{i+1, s}$ is not, then if $l(e, s)>x_{I, s}$, declare $x_{i, s}$ as active, and set $x_{i+1, s+1}=\mu z(z \geq s+2 \&(\exists k)(z=2 k))$. Set $\tilde{r}(e, s+1)=\max \left(u\left(x_{k, s}, e, s\right)\right.$ : $k \leq i)$. To get an idea of the restriction function $\tilde{r}(e, s)$, we give its definition, although it is not used in the basic module.

We say that $x_{i, s}$ is superactive, if $x_{i, s}-2$ and $x_{i, s}-1$ belong to $A_{s}$. Permission. If $x_{i, s}$ is active and $i \in B_{a t s}$, then if

(i) $(\exists j>i)\left[x_{j, s}\right.$ is superactive \& $\left.x_{j, s} \notin A_{s}\right]$, let $j_{0}=\mu z\left[x_{z, s}\right.$ is superactive \& $\left.x_{j, s} \notin A_{s}\right]$. Then we enumerate the numbers $x_{j_{0}, s}, \hat{x}_{j_{0}}, s$ into $A_{s+1}$ (where $\hat{x}_{j_{0}, s}=\psi_{e}\left(x_{j_{0}, s}\right)$ ). Cancel $x_{k, s}$, for all $\left(k>j_{0}\right)$. We will do the same with the accompanying elements of the corresponding followers.

(ii) if (i) and $(\neg \exists j)\left[x_{j, s} \in A_{s}\right]$ does not hold, then we enumerate the numbers $x_{i, s}-2, x_{i, s}-1$ into $A_{s+1}$. Cancel $x_{k, s}$, for all $(k>i)$. We will do the same with the accompanying elements of the corresponding followers.

For any $i$ such that the follower $x_{i, s}$ is not canceled at the end of the part "permission" of the basic module and is active, let's set $x_{i, s+1}=x_{i, s}$. We will do the same with the accompanying elements of the corresponding followers.

The "cancellation" rule, which is present in the proof of Theorem 4.8 (Downey, Slaman [8]), in this case it will be necessary to note the effect of the requirements of $R_{j}$ and $P_{j}$ (where $j<e$ ) on satisfying the requirement $R_{e}$, but not to describe the basic module itself for $R_{e}$. 


\subsection{Basic Module for $P_{e}$}

The followers $y_{1, s}, \ldots, y_{n, s}$ satisfy the following rules below.

Appointment. If $y_{i, s}$ is currently defined and $y_{i+1, s}$ is not, then if $(\exists z)\left(z \in W_{e} z \geq y_{i, s}\right)$, declare $y_{i, s}$ as active, and set $y_{i+1, s+1}=\mu z(z \geq s \&(\exists k)(z=2 k))$.

Permission. If $y_{i, s}$ is active and $i \in B_{a t s}$, then enumerate the numbers $y_{i, s}, y_{i, s}+1, z$ and $z+1$ into $A_{s+1}$.

The "cancellation" rule, which is present in the proof of Theorem 4.8 (Downey, Slaman [8]), in this case it will be necessary to note the effect of the requirements of $R_{j}$ (where $j \leq e$ ) on satisfying the requirement $P_{e}$, but not to describe the basic module itself for $P_{e}$.

\section{Verification of Lemmas}

Lemma 1: Suppose that $\psi_{e}$ is total and $(\forall x)\left(\Psi_{e}(A \cup\{x\} ; x) \downarrow\right)$.

Then $(\exists y) \neg\left(\left(\Psi_{e}(A \cup\{y\} ; y)=A(y)\right)\right.$. Thus, the requirement $R_{e}$ is satisfied.

Proof. Suppose otherwise. We show that $B$ is computable.

Note that since we only consider the satisfaction of the basis module for $R_{e}$ (that is, we do not take into account the effect of the requirements $R_{j}$ and $P_{j}$ (where $j<e$ ) on the satisfaction of the requirement $R_{e}$ ), it is obvious that conditions (i), ..., (iv) are met.

(i) All the $x_{i, s}$ eventually become permanently defined, that is $\lim _{s} x_{i, s}=x_{i}$ exists with $x_{i} \notin A$.

(ii) Once $x_{k}$ is defined at stage $t,(\forall s>t)\left(u\left(x_{k}, e, t\right)=u\left(x_{k}, e, s\right)=u\left(e, x_{k}\right)\right)$.

(iii) $(\forall i)\left(x_{i+1}>\max \left\{u\left(e, x_{k}\right): k \leq i\right\}\right)$.

(iv) It can be effectively recognized, when (i) occurs.

Two cases are possible:

(a) $(\exists m)(\forall k>m)\left[x_{k}-2 \notin A\right]$;

(b) $(\forall m)(\exists k>m)\left[x_{k}-2 \in A\right]$.

For both cases ((a) and (b)), it will be proved that $B$ is computable (and thus, the assumption that Lemma1 is false will lead to a contradiction with the supposition of noncomputability of $B$ ).

Now, if (a) holds, we prove that $B$ is computable.

If conditions (i), ..., (iv) are satisfied, we show how to compute $B$ (that is, the characteristic function of the set $B$; remind that we often identify the set $B$ with its characteristic function).

Let $f \| x$ denotes the restriction of $f$ to arguments $y<x$, and $A \| x$ denotes $\chi_{A} \| x$.

Let $s_{0}$ be such a stage that $B\left\|m+1=B_{s_{0}}\right\| m+1$ and $A\left\|x_{m+1}=A_{s_{0}}\right\| x_{m+1}$.

Let $q \in \omega$ and $q>m$. Effectively compute a stage $s$ so that $x_{q+1}$ is defined, that is $x_{q+1}=x_{q+1, s}$ (in that case, in fact, $s>s_{0}$ ).

Then $x_{q}$ is active, $x_{q} \in A$ and since $x_{q+1}$ is the final value of the $q+1$-th follower, the computations of $u\left(x_{j}, e, s\right)$ are true for all $j \leq q$.

In this case $q \in B \Leftrightarrow q \in B_{s}$, because otherwise it would lead to the fact that $x_{q}-2$ 
would have entered the set, contrary to our assumption that case (a) holds.

Now suppose that case (b) holds. Let us prove that in this case also $B$ is computable.

If conditions (i), .., (iv) are fulfilled, we show how to compute $B$.

Let $q \in \omega$. Effectively compute such a stage $s$ and a number $p$ so that $p=\mu z(z \geq q \&$ $\left.x_{z-2} \in A \& x_{z+1}=x_{z+1, s}\right)$.

Then $x_{p}$ is active, $x_{p} \notin A_{s}$ and since $x_{p+1}$ is the final value of the $p+1$-th follower, then $u\left(x_{j}, e, s\right)$ computations are true for all $j \leq p$. In this case $q \in B \Leftrightarrow q \in B_{s}$, since otherwise (that is, if $q$ enters $B$ after the stage $s$ ) this will lead to the entry $p$ into $A$ and satisfaction of the requirement $R_{e}$, which will contradict the initial assumption that Lemma 1 is false.

Lemma 1 is prooved.

Lemma 2: Suppose that $W_{e}$ is an infinite set. Then $(\exists z)\left(z \in W_{e} \& z \in A\right)$. Thus, the requirement $P_{e}$ is satisfied.

Proof. Suppose otherwise.

We show that $B$ is computable.

Let $\tilde{r}(e)=\lim _{s} \tilde{r}(e, s)$.

Although the use of this function in the description of the basis module for $P_{e}$ is not necessary, an indication of this function clearly shows the effect of the requirements $R_{j}$ (where $j \leq e$ ) on the satisfaction of the requirements $P_{e}$ when constructing the set $A$.

Let $t_{0}$ be such that $\left(\forall s \geq t_{0}\right) \tilde{r}(e, s)=\tilde{r}(e)$.

Then it is obvious, that all the $y_{i, s}$ become permanently defined (i.e., $\forall i \exists\left(t \geq t_{0}\right)(\forall s)\left(y_{i, t}=\right.$ $\left.y_{i, s}=y_{i}\right)$ ) with $y_{i} \notin A$.

In fact, if there existed $k$ such that $y_{k} \in A$, then, by construction, there would exist $z$ such that $z \in W_{e} \cap A$.

Assuming the opposite of the statement of the proposition, we show how $B$ can be computed. Let $q \in \omega$. Find $t \geq t_{0}$ such that $y_{q}$ is permanently defined. Then $q \in B \Leftrightarrow q \in B_{t}$, since otherwise $q$ 's entry into $B$ would meet $P_{e}$.

Lemma 2 is prooved.

\section{Conclusion}

Note that the coherence of constructions to satisfy the requirements $R_{e}$ and $P_{e}$ (for all $e$ ) is not difficult, since satisfying the requirements $R_{e}$ and $P_{e}$ (for all $e$ ) requires a finite number of steps. We also note that the indicated method of constructing the set $A$ (based on the constructions for the basic modules) will result in the set $A \cap \omega_{e v}$ being $T$-equivalent to the set $A \cap \omega_{\text {od }}$.

These remarks allow us to complete the proof of the theorem.

Note that it follows from the above theorem that below any noncomputable c.e. degree there is an infinite number of noncomputable c.e. degrees with the abovementioned property (since the degree $\boldsymbol{a}$ (mentioned in the theorem), containing a simple set, is a noncomputable c.e. degree). 


\title{
References
}

[1] R. I. Soare, Recursively Enumerable Sets and Degrees, Springer-Verlag, 1987.

[2] H. Rogers, Theory of Recursive Functions and Effective Computability, McGraw-Hill Book Company, 1967.

[3] R. G. Downey and M. Stob, "Splitting Theorems In Recursion Theory", Ann. Pure Appl. Logic, vol. 65, pp. 1-106, 1993.

[4] A. H. Lachlan, The Priority Method. I, Zeitschrift fr mathematische Logik und Grundlagen der Mathematik, vol.13, pp. 1-10, 1967.

[5] R. Ladner, "A complete mitotic recursively enumerable degree", Trans. Amer. Math. Soc., 184, pp. 497-507, 1973.

[6] R. Ladner and L. Sasso, "The weak truth-table degrees of recursively enumerable sets", Arch. Math. Logik Grundlang Grundlagen der Mathematik, vol. 8, pp. 429-448, 1975.

[7] M. Ingrassia, P-generecity for recursive enumerable degrees, $\mathrm{PhD}$. Thesis, University of Illinois, 1981.

[8] R. G. Downey and T. A. Slaman, "Completely Mitotic R. E. Degrees", Ann. Pure Appl. Logic, vol.41, pp. 119-152, 1989.

[9] E. J. Griffiths, Completely Mitotic Turing Degrees, Jump Classes and Enumeration Degrees, Ph.D. Thesis, University of Wisconsin-Madison, 1998.

[10] R. Ladner, "Mitotic Recursively Enumerable Sets", The Journal of Symbolic Logic, vol. 38, no. 2, pp. 199-211, 1973.

[11] B. A. Trakhtenbrot, "On autoreducibility" (in Russian), Dokl. Akad. Nauk SSSR, vol. 192, pp. 1224-1227, 1970.

Submitted 04.07.2019, accepted 22.11.2019.

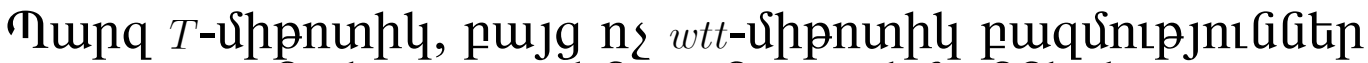

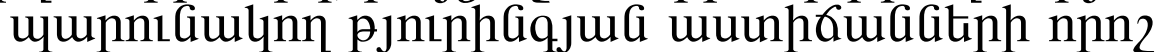 huulunıpjnıGiGiph ltipuptipjui}

\author{
Unutia ২. Unlumgjua

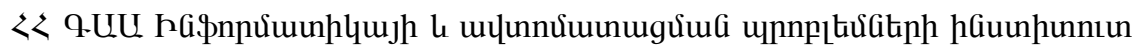 \\ e-mail: arsenmokatsian@gmail.com
}

\section{Uরuนnนhntư}

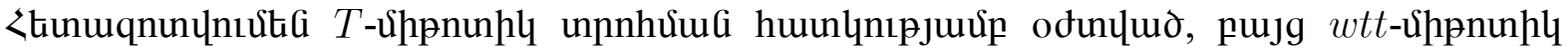

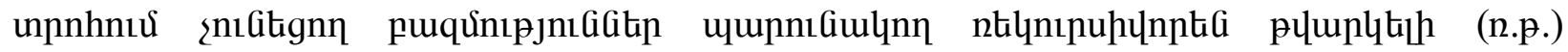

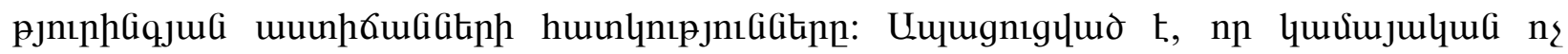

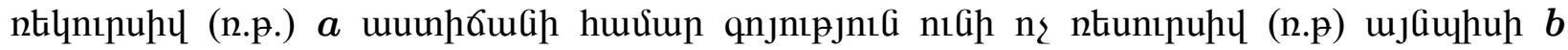

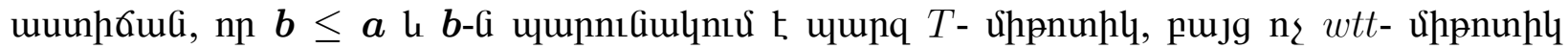
puquinıpjnıG: 


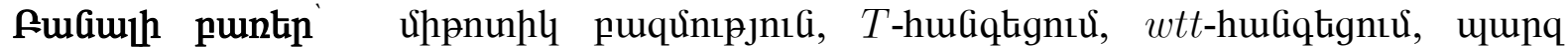
puqúnıpjnıG, qnıqulgцud uuunh6ua:

О некоторых свойствах тьюринговых степеней, содержащих простые $T$-митотические множества, не являющиеся $w t t$-Митотическими

\author{
Арсен А. Мокацян \\ Институт проблем информатики и автоматизации НАН РА \\ e-mail: arsenmokatsian@gmail.com
}

\begin{abstract}
Аннотация
Исследуются свойства рекурсивно перечислимых (р.п.) тьюринговых степеней, содержащих множества, которые обладают свойством -митотического разбиения, но не имеют $w t t$-митотического разбиения. Аоказано, что для любой нерекурсивной (р.п.) степени $\boldsymbol{a}$ существует нерекурсивная (р.п.) степень $\boldsymbol{b}$, такая что $\boldsymbol{b} \leq \boldsymbol{a}$ и $\boldsymbol{b}$ содержит простое $T$-митотическое множество, которое не является $w t t$-митотическим.

Ключевые слова: митотическое множество, T-сводимость, $w t t$-сводимость, простое множество, сцепленная степень.
\end{abstract}

